\title{
Comparative analysis of gene expression patterns in the arthropod labrum and the onychophoran frontal appendages, and its implications for the arthropod head problem
} CrossMark

Ralf Janssen * (1)

\begin{abstract}
The arthropod head problem has troubled scientists for more than a century. The segmental composition of the arthropod head, homology of its appendages, and especially the nature of the most anterior region of the head are still, at least partially, unclear. One morphological feature of the head that is in the center of current debate is the labrum (upper lip), a fleshy appendicular structure that covers the arthropod mouth. One hypothesis is that the labrum represents a fused pair of protocerebral limbs that likely are homologous with the frontal appendages (primary antennae) of extant onychophorans and the so-called great appendages of stem arthropods. Recently, this hypothesis obtained additional support through genetic data, showing that six3, an anterior-specific gene, is exclusively expressed in the arthropod labrum and the onychophoran frontal appendages, providing an additional line of evidence for homology. Here I present data that put this finding into perspective. The outcome of my study shows that the homologization of a morphological structure by the expression of a single genetic factor is potentially misleading.
\end{abstract}

Keywords: Arthropod head problem, Onychophora, Arthropoda, Labrum, Evolution

\section{Background}

"The endless dispute" [1] describes one of the most longstanding zoological problems: the segmental composition and homology of the anterior of the arthropod head and its appendages. Since Rempel's assumptions that these problems would likely never be solved, quite some progress has been made, and homology of head segments has been solved by the investigation of innervation patterns and Hox gene expression patterns (reviewed in [24]). A remaining problem, however, is the unclear nature of the pre-antennal (pre-cheliceral in Chelicerata) region, and the nature of the labrum, the upper lip of arthropods. The labrum represents an enigmatic structure of unclear origin and homology. It is discussed controversially if the labrum is an appendicular structure, and if it is serially

${ }^{*}$ Correspondence: ralf.janssen@geo.uu.se

Department of Earth Sciences, Palaeobiology, Uppsala University,

Villavägen 16, 75236 Uppsala, Sweden homologous to the other limbs. Even more unclear is its segmental affinity (if any) (reviewed in, e.g., $[1,3,5]$ ).

Most authors agree that the labrum represents a pair of fused limbs, based on the fact that it originates as two independent buds that fuse later during ontogeny, and partially conserved gene expression patterns (e.g., [6-15]). Nevertheless, some authors doubt its homology with the other limbs and instead suggest that the labrum may have evolved independently, and that the apparent genetic similarities are the result of convergent evolution rather than homology $[3,11-13,16,17]$.

Onychophorans represent the likely sister group to arthropods or at least form a closely related outgroup (depending on the still unclear phylogenetic position of Tardigrada) (e.g., [18-21]). Morphological studies and gene expression analysis of conserved Hox gene patterning in extant arthropods and onychophorans revealed that the onychophoran frontal appendages 
are not homologous with the mandibulate antennae (despite their morphological and functional similarity) but instead represent more anterior appendages [22-26]. Therefore, the idea has been put forward that the labrum is homologous with the frontal appendages of extant onychophorans and stem-group lobopodians and the great appendages of stem-group arthropods (e.g., [27$30])$, but see $[3,16,17]$ for another homology hypothesis.

In order to support homology of the frontal appendages with the labrum, Scholtz and Edgecombe [3] suggested to search for genes that are exclusively expressed in both structures, but not in any of the other appendages.

Recently, one such factor, six 3 , was revealed. six3 controls patterning of the so-called anterior median region (AMR) in bilaterian animals ([31-37]), and many of the genes that are expressed in the AMR are also expressed in the arthropod labrum offering the opportunity to find more shared specific factors that could support homology of the labrum with the frontal appendages.

In order to put the homology test (as suggested by [3]) to the test, in the current paper, expression patterns of AMR/labrum-patterning genes have been investigated in the onychophoran Euperipatoides kanangrensis and, in order to obtain a more solid basis for comparison, also in the myriapod Glomeris marginata. The data indicate that the AMR is principally conserved in onychophorans, but the ambiguous nature of the results with respect to expression in the frontal appendages indicates that homologization of the labrum with the frontal appendages based on the expression of a single gene, such as six 3 , is problematic.

\section{Methods}

\section{Embryo collection, fixation and developmental staging}

Onychophoran embryos were dissected from pregnant females, prepared for in situ hybridization experiments, and staged as described in [38, 39]. Myriapod embryos were collected, fixed and staged as described in [40].

\section{Gene cloning}

Total RNA was extracted (TRIzol, Invitrogen, Carlsbad, $\mathrm{CA}$ ) and reverse transcribed into cDNA (SuperScriptII first-strand synthesis system for RT-PCR, Invitrogen). Gene fragments were isolated by means of RT-PCR with gene-specific primers (Additional file 1: Table S1) based on sequenced embryonic transcriptomes. All gene fragments were cloned into pCRII vectors (TA cloning kit dual promoter, Invitrogen) and sequenced by a commercial sequencing service (Macrogen, Seoul, South Korea). Newly recovered sequences are available under accession nos. LT560250 (Ek-hbn), LT560249 (Ek-FoxQ2), LT560252 (Ek-nkx2.1/scro), LT560251 (Ek-rx), LT560253 $(E k-v s x / c h x), \quad$ LT560255 (Gm-hbn), LT560254 (GmFoxQ2), LT560256 (Gm-nkx2.1/scro).

\section{In situ hybridization}

Whole-mount in situ hybridization of E. kanangrensis and G. marginata embryos was performed as in [41]. Digoxigenin-labeled RNA probes were transcribed from the cloned gene fragments. Cell nuclei were stained by incubation in $2 \mu \mathrm{g} / \mathrm{ml}$ of the fluorescent dye 4-6-diamidino-2-phenylindole (DAPI) in phosphate-buffered saline with $0.1 \%$ Tween 20 (PBST) for 20-30 min at room temperature (Additional file 2: Table S2).

\section{Data documentation}

Photographs were taken with a Leica DC100 digital camera under a Leica dissection microscope. The image processing software Adobe PHOTOSHOP CS2 (v. 9.0.1 for Apple Macintosh) was used for linear corrections of brightness, contrast and color values.

\section{Phylogenetic analysis}

Protein sequences of the complete open reading frames of the homeodomain-encoding genes $\mathrm{Rx}, \mathrm{Vsx} / \mathrm{Chx}, \mathrm{Hbn}$ and Nkx2.1/Scro of the fly Drosophila melanogaster, the beetle Tribolium castaneum, Euperipatoides and Glomeris, have been aligned using T-Coffee [42]. As closely related outgroup sequences serve ventral nervous system defected (Vnd) from Drosophila and Tribolium as well as aristaless (Al) from Drosophila. The same has been done for the forkhead domain of all known Drosophila and Strigamia maritima Fox genes [43, 44] and FoxQ2 from Glomeris and Euperipatoides. The Fox2 gene of Saccharomyces cerevisiae serves as outgroup sequence. In both cases, Bayesian phylogenetic analyses were performed with MrBayes [45] using a fixed WAG amino acid substitution model with gammadistributed rate variation across sites (with four rate categories). An unconstrained exponential prior probability distribution on branch lengths and an exponential prior for the gamma shape parameter for among-site rate variation were applied. The final topology was estimated using 1,000,000 cycles for the MCMCMC (metropolis-coupled Markov chain Monte Carlo) analysis with four chains and the chain heating temperature set to 0.2 . The Markov chain was sampled every 200 cycles. Clade support was assessed with posterior probabilities computed with MrBayes.

\section{Results \\ Phylogenetic analysis}

Predicted onychophoran orthologs of visual system homeobox (Vsx/Chx), retinal homeobox (Rx), Scarecrow (Scro), homeobrain ( $\mathrm{Hbn}$ ) and FoxQ2 cluster with high reliability with their arthropod orthologs (Fig. 1a, b). The putative ortholog of ventral nervous system defective (Vnd) branches at the base of arthropod Vnd and arthropod + onychophoran Scro. Expression analysis revealed that Euperipatoides vnd is not expressed in the head, but 


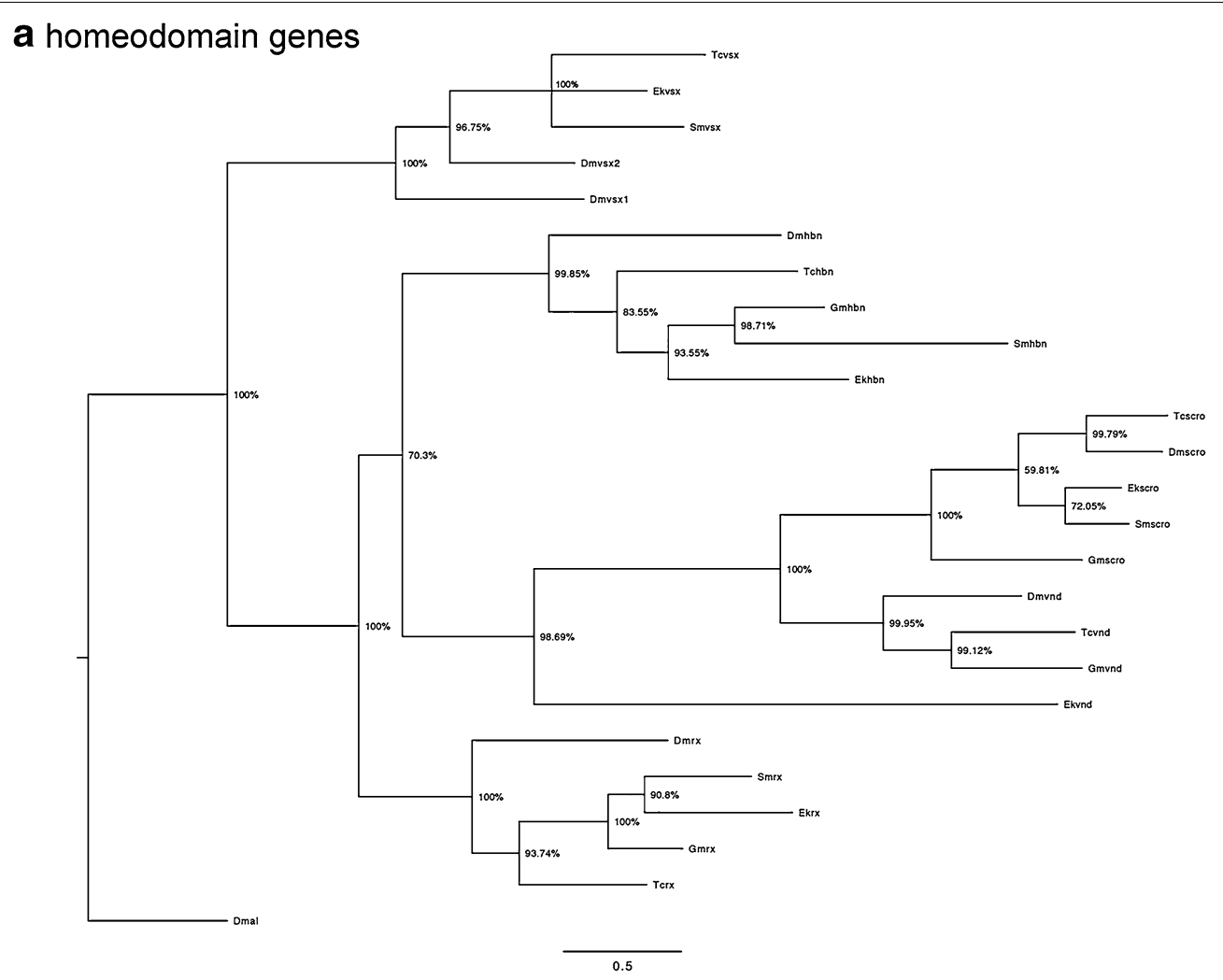

\section{b FoxQ2}

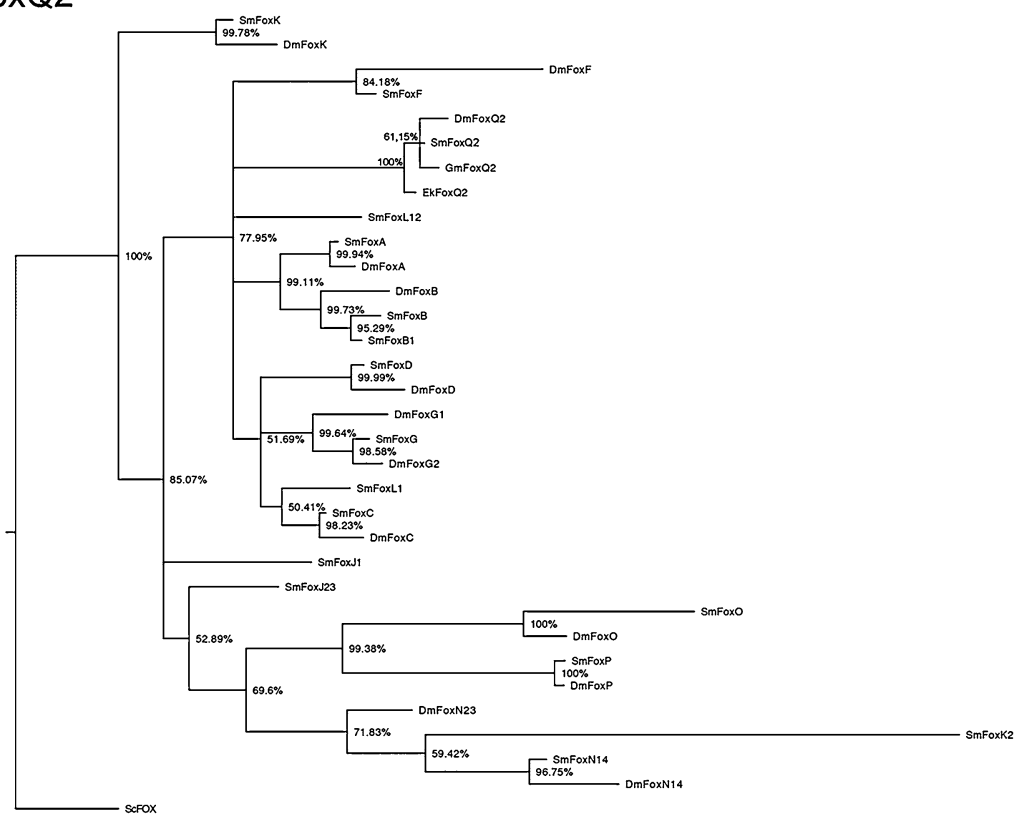

0.2

Fig. 1 Phylogenetic analysis of homeodomain genes and FoxQ2. Species abbreviations: Ek, Euperipatoides kanangrensis (Onychophora); Dm, Drosophila melanogaster (Hexapoda: Diptera); Gm, Glomeris marginata (Myriapoda: Diplopoda) Tc, Tribolium castaneum (Hexapoda: Coleoptera); Sm, Strigamia maritima (Myriapoda: Chilopoda). Gene abbreviations: al, aristaless; Fox, forkhead box gene; rx, retinal homeobox; scro, scarecrow; vnd, ventral nervous system defective; vsx, visual system homeobox. See text for further information 
is, like arthropod vnd genes, predominantly expressed in the ventral nerve cord (not shown).

\section{Expression patterns}

Euperipatoides homeobrain (Ek-hbn) is exclusively expressed in the head, including the developing frontal appendages. The latter is restricted to ventral and proximal tissue (Fig. 2), very similar to the expression of six3
[5]. Glomeris hbn is expressed in form of two patches in the very anterior of the head and in a domain in the primordium of the labrum (Fig. 2). At later stages, this latter expression is located dorsally in the developing labrum and thus in a comparable pattern as seen in the onychophoran (if the rotation theory put forward by [10] holds true).

Euperipatoides FoxQ2 (Ek-FoxQ2) is expressed in the ventral region of the head lobes, anterior to the mouth,

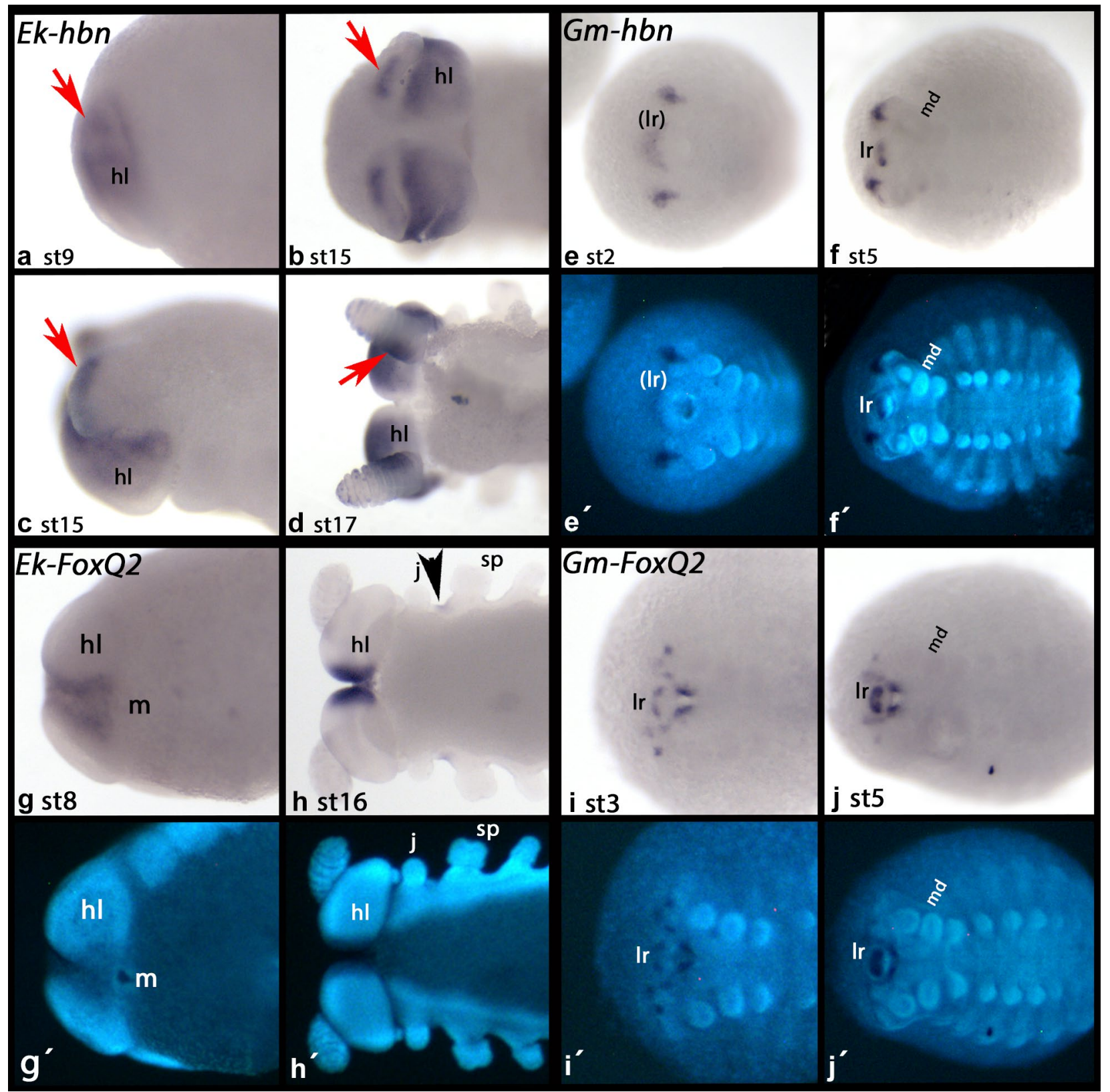

Fig. 2 Expression of $h b n$ and FoxQ2. In all panels anterior is to the left. Ventral view, if not indicated otherwise. a-d Euperipatoides hbn. a, c Lateral view. d Dorsal view. Arrows point to expression in the frontal appendages. e, f Glomeris hbn. $\mathbf{g}, \mathbf{h}$ Euperipatoides FoxQ2. Arrowhead marks expression between the jaw and the slime papilla. $\mathbf{i}, \mathbf{j}$ Glomeris FoxQ2. Ir primordium of the labrum, $h /$ head lobe, $j$ jaw, I labrum, $m$ mouth, $m d$ mandible, $s p$ lime papilla 
but not the frontal appendages. At later stages, it is expressed also in a patch-like domain at the base between the jaws and the slime papillae (Fig. 2). Glomeris FoxQ2 is expressed in a complex pattern in the anterior of the head including the labrum.

Euperipatoides scarecrow $(E k-n k x 2.1 /$ scro $)$ is expressed in ventral and posterior tissue of the head lobes, but not in the frontal appendages (Fig. 3). In contrast to that, Glomeris $n k x 2.1 /$ scro is expressed in a complex pattern in the anterior head as well as in the labrum (Fig. 3).

Euperipatoides retinal homeobox (Ek-rx) is exclusively expressed in the head lobes. At early developmental stages, it is expressed weakly and transiently in the frontal appendages. Later, however, it is not expressed in the frontal appendages anymore (Fig. 3).

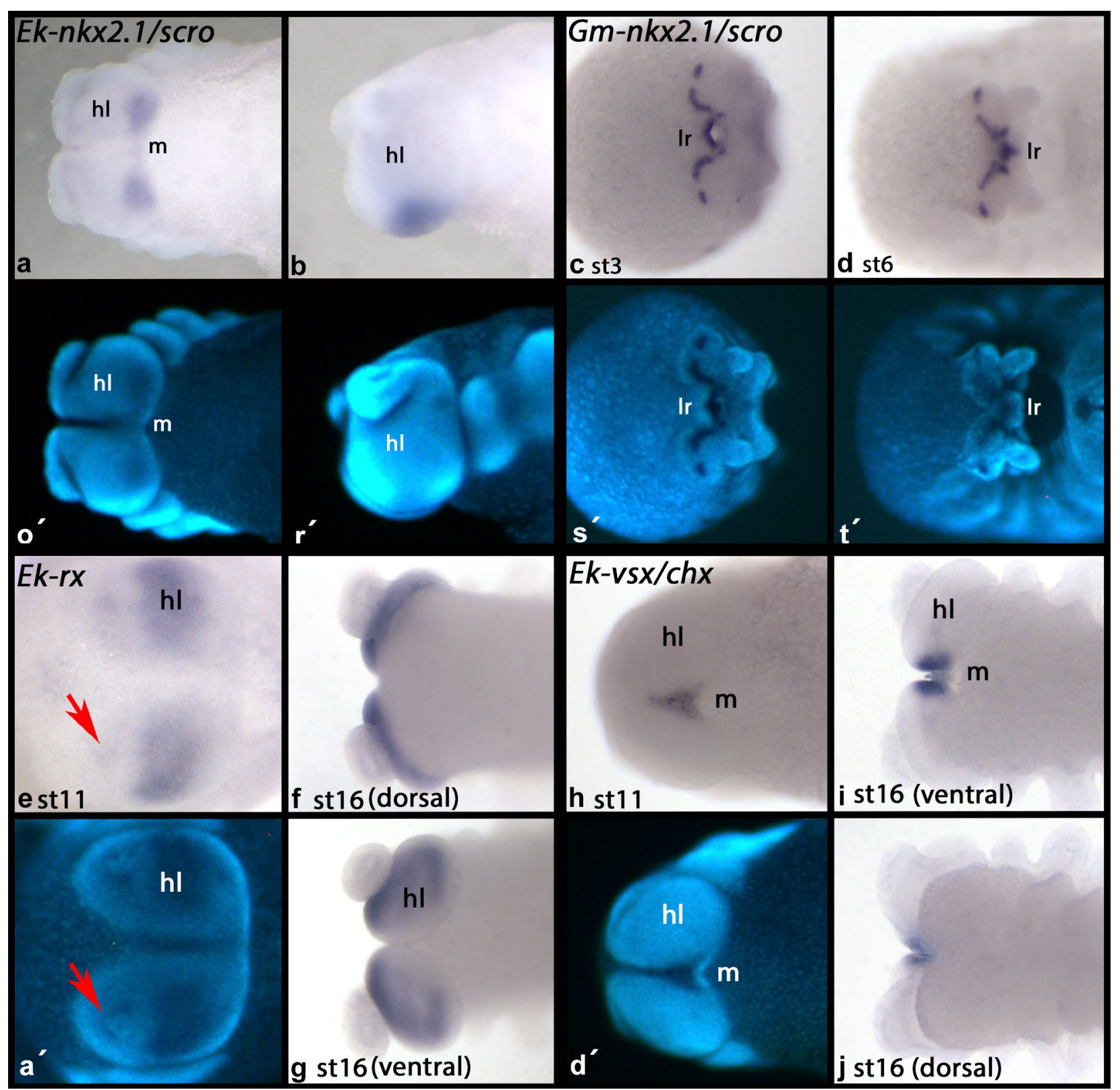

Fig. 3 Expression of $n k x 2.1 / s c r o, r x$ and $v s x / c h x$. In all panels anterior is to the left. Ventral view, if not indicated otherwise. a, b Euperipatoides $n k x 2.1 /$ scro. b Lateral view. c, d Glomeris $n k x 2.1 /$ scro. c Anterior view. e-g Expression of Euperipatoides rx. a anterior view. h-j Expression of Euperipatoides vsx/chx. j Dorsal view. Abbreviations as in Fig. 2 
Euperipatoides visual system homeobox (Ek-vsx/chx) is expressed in the very ventral region of the head lobes, anterior of the mouth. This domain resembles very much the domain in which FoxQ2 is expressed. However, expression of $v s x / \operatorname{ch} x$ is slimmer and does not reach as far toward anterior as that of FoxQ2. Also, $v s x /$ chx is not expressed posterior to the head lobes (Fig. 3).

\section{Discussion}

\section{Putting the homology test to the test: taking a look beyond six 3}

In their very much noticed review article Scholtz and Edgecombe [3] suggested to search for genes that are specifically expressed in the labrum and the frontal appendages (but not in any other appendage type) since those patterns would likely serve as direct evidence for homology: "..., if peculiarities of gene expression in the labrum, which are absent in trunk limbs of euarthropods, find their correspondence in that of onychophoran 'antennae,' we could have direct evidence for homology between these two structures" [3].

Possibly as a direct consequence, when the test was undertaken, as expression of six 3 was found in both, the arthropod labrum and the onychophoran frontal appendages, some authors took this as a possible, or even convincing, homology criterion [5, 23, 46, 47].

However, the past has shown that it is problematic to use a single gene as strong evidence for homology (discussed in [48]), as exemplified by the use of, e.g., the segment polarity gene engrailed (en) alone and out of context for the identification of head segments (discussed in, e.g., [4]), or the use of distal-less (Dll) alone, and out of its genetic context, for the identification of serially homologous appendages (discussed in, e.g., [9]). $e n$ and $D l l$ are embedded in a conserved gene regulatory network as segmentation and appendage-patterning genes, respectively, but they are also expressed in other, non-homologous structures such as anal valves and sensory bristles. The same problem may occur with the use of six3 as a marker for the labrum/frontal appendages. In fact, the role of six3 in appendage patterning is not exclusive for the frontal appendages of onychophorans and the arthropod labrum, but it is also expressed in other sensory appendages in at least myriapods [33,36], although this may be explained best by convergent evolution rather than an ancestral role of six 3 in patterning these limbs.

However, as Aristotle (384-322 BC) pointed out "One swallow does not a summer make, ..., likewise the expression of one gene, not even that of the famous six3, Dll, or en necessarily defines the evolutionary nature of a morphological structure.

The data obtained in this study support this concern as they are ambiguous (Figs. 2, 3). While some genes appear to support homology of the arthropod labrum with the onychophoran frontal appendages (i.e., six3 and $h b n$ ) (Fig. 2) [5, 37], others do not, since they are not expressed in the frontal appendages although their arthropod orthologs are expressed in the labrum (i.e., $r x$, scro, FoxQ2, vs $x / \operatorname{ch} x$ ) (Figs. 2, 3) [34, 37]. This does not only impede interpretation, but also cast doubts on the suitability of anteriorly expressed markers for the testing of the homology hypothesis concerning the labrum and the frontal appendages.

It remains unclear whether the observed differences in the onychophoran and/or arthropod lineage are a result of morphological change during the course of evolution, or whether the similar expression patterns in the labrum and the frontal appendages merely are the result of convergent evolution and the recruitment of a similar set of genes or a remnant of homology. We have to understand the function and the gene regulatory network in which six3 is involved better to make convincing statements about its potential relevance as homology criterion.

\section{Conclusions}

This study shows that the gene expression landscape in the anterior head of onychophorans and arthropods does not unambiguously support homology of the arthropod labrum with the frontal appendages (=primary antennae) of onychophorans. Instead, the new data reveal that only some of the genes that are expressed in the labrum are also expressed in the frontal appendages. Thus, it is not justified to take a single gene that is expressed in both structures as strong evidence for homology.

\section{Additional files}

Additional file 1: Table S1. Primer sequences.

Additional file 2: Table S2. Accession numbers for genes used in the phylogenetic analysis.

\section{Acknowledgements}

I gratefully acknowledge the support of the New South Wales Government Department of Environment and Climate Change by provision of a permit SL100159 to collect onychophorans at Kanangra-Boyd National Park. I thank Glenn Brock, David Mathieson, Robyn Stutchbury and especially Noel Tait, for their help during onychophoran collection.

\section{Competing interests}

The author declares that he has no competing interests.

\section{Data availability}

All data underlying the current analyses are publicly available or are included in the supplementary files.

\section{Funding}

This work has been supported by the Swedish Research Council (VR).

Received: 6 November 2016 Accepted: 15 December 2016

Published online: 03 January 2017 


\section{References}

1. Rempel JG. The evolution of the insect head: the endless dispute. Q Entomol. 1975:11:7-25.

2. Hughes $\mathrm{CL}$, Kaufman TC. Hox genes and the evolution of the arthropod body plan. Evol Dev. 2002;4:459-99.

3. Scholtz G, Edgecombe GD. The evolution of arthropod heads: reconciling morphological, developmental and palaeontological evidence. Dev Genes Evol. 2006;216:395-415

4. Bitsch J, Bitsch C. The tritocerebrum and the clypeolabrum in mandibulate arthropods: segmental interpretation. Acta Zool. 2010;91:249-66.

5. Ortega-Hernández J, Janssen R, Budd GE. Origin and evolution of the panarthropod head - a palaeobiological and developmental perspective. Arthropod Struct Dev. 2016. doi:10.1016/j.asd.2016.10.011

6. Abzhanov A, Kaufman TC. Homologs of Drosophila appendage genes in the patterning of arthropod limbs. Dev Biol. 2000;227:673-89.

7. Prpic NM, Wigand B, Damen WG, Klingler M. Expression of dachshund in wild-type and Distal-less mutant Tribolium corroborates serial homologies in insect appendages. Dev Genes Evol. 2001:211:467-77.

8. Prpic NM, Janssen R, Wigand B, Klingler M, Damen WG. Gene expression in spider appendages reveals reversal of exd/hth spatial specificity, altered leg gap gene dynamics, and suggests divergent distal morphogen signaling. Dev Biol. 2003:264:119-40

9. Prpic NM, Tautz D. The expression of the proximodistal axis patterning genes Distal-less and dachshund in the appendages of Glomeris marginata (Myriapoda: Diplopoda) suggests a special role of these genes in patterning the head appendages. Dev Biol. 2003;260:97-112.

10. Kimm MA, Prpic NM. Formation of the arthropod labrum by fusion of paired and rotated limb-bud-like primordia. Zoomorphology. 2006:125:147-55.

11. Posnien N, Bashasab F, Bucher G. The insect upper lip (labrum) is a nonsegmental appendage-like structure. Evol Dev. 2009;11:480-8.

12. Posnien N, Schinko JB, Kittelmann S, Bucher G. Genetics, development and composition of the insect head - a beetle's view. Arthropod Struct Dev. 2010;39:399-410

13. Liu Y, Maas A, Waloszek D. Early development of the anterior body region of the grey widow spider Latrodectus geometricus Koch, 1841 (Theridiidae, Araneae). Arthrop Struct Dev. 2009;38:401-16.

14. Liu Y, Maas A, Waloszek D. Early development of the head region of Gryllus assimilis Fabricius, 1775 (Orthoptera, Insecta). Arthropod Struct Dev. 2010;39:382-95.

15. Janssen R, Posnien N. Identification and embryonic expression of Wnt2, Wnt4, Wnt5 and Wnt9 in the millipede Glomeris marginata (Myriapoda: Diplopoda). Gene Expr Patterns. 2014;14:55-61.

16. Frase T, Richter $\mathrm{S}$. The fate of the onychophoran antenna. Dev Genes Evol. 2013;223:247-51

17. Scholtz G. Perspective-heads and brains in arthropods: 40 years after the "Endless Dispute". In: Schmidt-Rhaesa A, Harzsch S, Purschke G, editors. Structure and evolution of invertebrate nervous systems. Oxford: Oxford University Press; 2016. p. 402-10

18. Campbell LI, Rota-Stabelli O, Edgecombe GD, Marchioro T, Longhorn SJ, Telford MJ, Philippe H, Rebecchi L, Peterson KJ, Pisani D. MicroRNAs and phylogenomics resolve the relationships of Tardigrada and suggest that velvet worms are the sister group of Arthropoda. Proc Natl Acad Sci USA. 2011;108:15920-4.

19. Rota-Stabelli O, Campbell L, Brinkmann H, Edgecombe GD, Longhorn SJ, Peterson KJ, Pisani D, Philippe H, Telford MJ. A congruent solution to arthropod phylogeny: phylogenomics, microRNAs and morphology support monophyletic Mandibulata. Proc Biol Sci. 2011;278:298-306.

20. Borner J, Rehm P, Schill RO, Ebersberger I, Burmester T. A transcriptome approach to ecdysozoan phylogeny. Mol Phylogenet Evol. 2014;80:79-87.

21. Smith MR, Ortega-Hernández J. Hallucigenia's onychophoran-like claws and the case for Tactopoda. Nature. 2014:514:363-6.

22. Eriksson BJ, Budd GE. Onychophoran cephalic nerves and their bearing on our understanding of head segmentation and stem-group evolution of Arthropoda. Arthropod Struct Dev. 2000;29:197-209.

23. Eriksson BJ, Tait NN, Budd GE. Head development in the onychophoran Euperipatoides kanangrensis with particular reference to the central nervous system. J Morphol. 2003;255:1-23.

24. Whitington $P M$, Mayer $G$. The origins of the arthropod nervous system: insights from the Onychophora. Arthropod Struct Dev. 2011:40:193-209.
25. Eriksson BJ, Tait NN, Budd GE, Janssen R, Akam M. Head patterning and Hox gene expression in an onychophoran and its implications for the arthropod head problem. Dev Genes Evol. 2010;220:117-22.

26. Janssen R, Eriksson BJ, Tait NN, Budd GE. Onychophoran Hox genes and the evolution of arthropod Hox gene expression. Front Zool. 2014;11:22.

27. Budd GE. A palaeontological solution to the arthropod head problem. Nature. 2002:417:271-5

28. Cong P, Ma X, Hou X, Edgecombe GD, Strausfeld NJ. Brain structure resolves the segmental affinity of anomalocaridid appendages. Nature. 2014;513:538-42

29. Chipman AD. An embryological perspective on the early arthropod fossil record. BMC Evol Biol. 2015;15:285.

30. Ortega-Hernández J, Budd GE. The nature of non-appendicular anterior paired projections in Palaeozoic total-group Euarthropoda. Arthropod Struct Dev. 2016:45:185-99.

31. Oliver G, Mailhos A, Wehr R, Copeland NG, Jenkins NA, et al. Six3, a murine homologue of the sine oculis gene, demarcates the most anterior border of the developing neural plate and is expressed during eye development. Development. 1995;121:4045-55.

32. Coiffier D, Charroux B, Kerridge S. Common functions of central and posterior Hox genes for the repression of head in the trunk of Drosophila. Development. 2008;135:291-300.

33. Steinmetz PR, Urbach R, Posnien N, Eriksson J, Kostyuchenko RP, Brena C, Guy K, Akam M, Bucher G, Arendt D. Six3 demarcates the anterior-most developing brain region in bilaterian animals. EvoDevo. 2010;1:14.

34. Posnien N, Koniszewski ND, Hein HJ, Bucher G. Candidate gene screen in the red flour beetle Tribolium reveals six3 as ancient regulator of anterior median head and central complex development. PLOS Genet. 2011:12:e1002416.

35. Eriksson BJ, Samadi L, Schmid A. The expression pattern of the genes engrailed, pax6, otd and six3 with special respect to head and eye development in Euperipatoides kanangrensis Reid 1996 (Onychophora: Peripatopsidae). Dev Genes Evol. 2013:223:237-46.

36. Janssen R. Developmental abnormalities in Glomeris marginata (Villers 1789) (Myriapoda: Diplopoda): implications for body axis determination in a myriapod. Naturwissenschaften. 2013;100:33-43.

37. HunnekuhI VS, Akam M. An anterior medial cell population with an apical-organ-like transcriptional profile that pioneers the central nervous system in the centipede Strigamia maritima. Dev Biol. 2014;396:136-49.

38. Janssen R, Eriksson BJ, Budd GE, Akam M, Prpic NM. Gene expression patterns in an onychophoran reveal that regionalization predates limb segmentation in pan-arthropods. Evol Dev. 2010;12:363-72.

39. Janssen R, Budd GE. Deciphering the onychophoran'segmentation gene cascade': gene expression reveals limited involvement of pair rule gene orthologs in segmentation, but a highly conserved segment polarity gene network. Dev Biol. 2013;382:224-34.

40. Janssen R, Prpic NM, Damen WG. Gene expression suggests decoupled dorsal and ventral segmentation in the millipede Glomeris marginata (Myriapoda: Diplopoda). Dev Biol. 2004;268:89-104.

41. Janssen R, Jörgensen M, Pripic NM, Budd GE. Aspects of dorso-ventral and proximo-distal limb patterning in onychophorans. Evol Dev. 2015;17:21-33.

42. Notredame C, Higgins DG, Heringa J. T-Coffee: a novel method for fast and accurate multiple sequence alignment. J Mol Biol. 2000;302:205-17.

43. Chipman $A D$, et al. The first myriapod genome sequence reveals conservative arthropod gene content and genome organisation in the centipede Strigamia maritima. PLoS Biol. 2014;12:e1002005.

44. Kenny NJ, Shen X, Chan TT, Wong NW, Chan TF, Chu KH, Lam HM, Hui JH. Genome of the rusty millipede, Trigoniulus corallinus, illuminates diplopod, myriapod, and arthropod evolution. Genome Biol Evol. 2015;7:1280-95.

45. Huelsenbeck JP, Ronquist F. MRBAYES: bayesian inference of phylogenetic trees. Bioinformatics. 2001:17:754-5.

46. Ortega-Hernández J. Homology of head sclerites in Burgess Shale euarthropods. Curr Biol. 2015;25:1625-31.

47. Ortega-Hernández J, Budd GE. The nature of non-appendicular anterior paired projections in Palaeozoic total-group Euarthropoda. Arthropod Struct Dev. 2016:45:185-99.

48. Scholtz G. Evolution of developmental patterns in arthropods-the contribution of gene expression to morphology and phylogenetics. Zoology. 2001;103:99-111. 